\title{
Testing for Depressive Realism in a Clinically Depressed Sample
}

\author{
Shruti Venkatesh,' Michelle L. Moulds' and Christopher J. Mitchell ${ }^{2}$ \\ I The University of New South Wales, Sydney, New South Wales, Australia \\ 2 Plymouth University, Devon, UK
}

\begin{abstract}
The depressive-realism effect refers to a phenomenon in which depressed individuals are more realistic at assessing the relationship between two events than non-depressed individuals. Recent evidence suggests that the depressive realism hypothesis is weaker than first thought. Thus, we sought evidence for depressiverealism under conditions that we hypothesised would maximise the effect. We tested a clinically depressed sample of participants who were administered a rumination induction. Twenty-eight clinically depressed and 39 non-depressed participants were randomly allocated to either a rumination condition (focused on the causes, consequences, and meaning of their mood) or a distraction condition (focused on external objects/events such as a classroom). Participants then completed a contingency task in which there was no relationship between their responses and an outcome, and they were asked to make a judgment of how much control they had over an outcome. Both groups and conditions did not differ in their judgments of control; participants in all conditions showed a non-normative judgment of control. The depressive-realism effect was not observed in this study, even when depressed participants were encouraged to ruminate. Rather, the present study clearly demonstrates the robustness of the illusion of control.
\end{abstract}

Keywords: depression, associative learning, attention, judgment, rumination

Depressive realism was first demonstrated by Alloy and Abramson (1979) in a study of contingency learning, which involves learning the relationship between two (or more) events; for example, learning that a particular action or response (e.g., pressing a button) leads to the occurrence of an outcome (e.g., onset of a light). In a contingency task, the individual can choose to make a response $(\mathrm{R})$ with the outcome either appearing $(\mathrm{O}$; represented by the a cell) or not appearing $(\sim \mathrm{O}$; represented by the $\mathrm{b}$ cell) on that trial. Alternatively, the individual may choose to withhold their response $(\sim R)$, with the outcome either appearing (represented by the c cell) or not appearing (represented by the $\mathrm{d}$ cell) on that trial. The combination of the frequencies of these four events (or cells) can be used to calculate the objective contingency between the response and the outcome.

Alloy and Abramson (1979) sought to investigate whether individuals' perceptions of contingencies correspond to the way in which objective contingency $(\Delta P)$ is calculated. They employed a contingency task in which participants were asked to provide a judgment of how much control they thought they had over the illumination of a light (the outcome). Participants could test their degree of control by choosing

\footnotetext{
Address for correspondence: Shruti Venkatesh, School of Psychology, University of New South Wales, Sydney NSW 2052, Australia.

Email: shrutivenkatesh@outlook.com
}

Behaviour Change I Volume 35 I Number 2 | 2018 | pp. 108-122 I C) The Author(s) 2018 doi 10.1017/bec.2018.12 
to press a button (the response) over a series of trials. Participants' judgment of control was then compared to the objective contingency between the response and the outcome (set by the experimenter using $\Delta \mathrm{P}$ ) to determine how accurate participants were in their assessment of the contingency. $\Delta \mathrm{P}$ is calculated in the following manner (Allan, 1980):

$$
\text { Delta } \mathrm{P}=\mathrm{P}(\mathrm{O} / \mathrm{R})-\mathrm{P}(\mathrm{O} / \sim \mathrm{R})=\mathrm{a} /(\mathrm{a}+\mathrm{b})-\mathrm{c} /(\mathrm{c}+\mathrm{d})
$$

Alloy and Abramson (1979) were interested in examining depressed and nondepressed ${ }^{1}$ participants' judgments of control, in which the response and the outcome were independent (i.e., zero contingency task). They hypothesised that depressed individuals would either underestimate the objective contingency between their responses and the outcome or underestimate contingency relative to non-depressed participants. Non-depressed individuals overestimated their degree of control and illustrated an illusion of control; that is, they believed that they had control over the illumination of the light even when they did not. However, depressed individuals were significantly more accurate in judging the contingency; that is, their judgments of control were closer to zero than were those of the non-depressed participants (although their ratings were not precisely zero). In other words, depressed individuals did not succumb to the illusion of control. This effect came to be referred to in the literature as the depressive realism effect.

A recent meta-analytic review conducted by Moore and Fresco (2012) reported that the effect size of the depressive realism effect was Cohen's $d=-.07$, which represents a small conventional effect size. Rather, Moore and Fresco found that depressed and non-depressed participants overestimated their degree of control to the same extent (Cohen's $d=.53$ and .60 respectively). Thus, participants tend to display a robust illusion of control. Although the depressive realism effect is small, it is critical to replicate the effect in order to understand what appears to be a complex relationship between depressive realism and depression — and particularly because the effect appears to be inconsistent with current theoretical understanding of depression. Specifically, the negative distorted thinking styles that are characteristic of individuals with depression (Beck, 1967; Beck, Rush, Shaw, \& Emery, 1979) are at odds with the purported realism displayed in the depressive realism effect. In order to maximise the probability of replicating this effect, we consider it important to capitalise on conditions that are theorised to account for the effect.

One major theory proposed to account for the depressive realism effect is the intertrial interval (ITI) hypothesis (Msetfi, Murphy, \& Simpson, 2007; Msetfi, Murphy, Simpson \& Kornbrot, 2005). The ITI hypothesis suggests that the depressive realism effect is due to the way in which individuals perceive and process the time between trials. In particular, Msetfi et al. (2005) suggested that the ITI could be perceived to be similar to when a person chooses not to make a response and no outcome follows (i.e., ' $d$ ' cell). If the individual perceives the ITI as d cells, and they integrate them into their contingency calculation, this would effectively lead to an increase in the perceived contingency. When the contingency between the response and outcome is zero, integration of ITIs into the contingency calculation would lead to a significant increase in contingency. Msetfi et al. (2005) proposed that non-depressed individuals integrate the ITI into their judgments, thereby exhibiting elevated judgments of control (i.e., an illusion of control). Depressed individuals, on the other hand, do not integrate the ITI into their judgments because of their tendency to engage in rumination, which 
may occur during the ITI period, thereby avoiding inflated contingency judgments, hence exhibiting the depressive realism effect.

Rumination is a key cognitive factor linked to the maintenance of depression and a type of maladaptive attentional focus in which depressed individuals dwell on the causes, consequences and meanings of their depressive symptoms. In studies that have investigated the effects of rumination on cognitive processes, researchers have administered a rumination manipulation and compared the downstream consequences to those that follow a distraction condition (e.g., Nolen-Hoeksema, Morrow, $\&$ Fredrickson, 1993). Following rumination (as opposed to distraction), high dysphoric individuals demonstrated poorer social problem solving (e.g., Lyubomirsky \& Nolen-Hoeksema, 1995) and recalled fewer specific details of autobiographical memories (e.g., Lyubomirsky, Caldwell, \& Nolen-Hoeksema, 1998). Thus, rumination (particularly abstract rumination - a subtype of depressive rumination) has maladaptive consequences for high dysphoric and clinically depressed individuals (e.g., Donaldson \& Lam, 2004; Watkins \& Moulds, 2005).

In the typical design of experimental studies in the rumination literature, in one condition participants are instructed to focus on the meanings of their experience (i.e., the rumination condition), and in the comparison condition they are instructed to focus on external events (i.e., the distraction condition). In the rumination condition, participants are told to 'think about the causes, consequences and meaning' of a range of statements about their current mood and internal states; for example, 'physical sensations in your body' (Watkins \& Teasdale, 2004). In the distraction condition, participants are instructed simply to think about a series of external items; for example, 'imagine a boat slowly crossing the Atlantic' (Nolen-Hoeksema et al., 1993). Following the manipulations, participants rate the extent to which they were focused on their internal state and the degree to which they were focused on meanings of experiences. As a result of an effective rumination manipulation, participants report greater selffocus and greater focus on the meanings of the items as compared to participants in the distraction condition.

One way to maximise the replication of the depressive realism effect and test the ITI hypothesis would be to experimentally manipulate rumination and examine whether this has an impact upon judgments of control. If rumination influences judgements of control it may be a major determinant of the depressive realism effect. Msetfi et al. (2005) tested the ITI hypothesis and found that the length of the ITI affected non-depressed but not depressed individuals' judgement of control, such that the depressive realism effect occurred when the ITI was long but not short. They concluded that depressed individuals must be engaged in the process of rumination and thereby not perceive the ITI as a 'd' cell. Given that clinically depressed individuals characteristically engage in rumination (Nolen-Hoeksema, 1991), we consider it plausible that this quantitative difference in cognitive and reasoning processes relative to non-depressed individuals and dysphoric individuals may lead to stronger depressive realism and accuracy in a contingency task. Accordingly, we conducted a replication of this study using a clinically depressed sample.

We tested the ITI hypothesis by investigating the consequences of rumination (relative to distraction) for judgments of control in clinically depressed individuals. Consistent with the ITI hypothesis, we hypothesised that clinically depressed participants in the rumination condition would be more accurate than those in the distraction condition. The rumination manipulation has negative cognitive and reasoning consequences for clinically depressed individuals but not for non-depressed 
individuals (e.g., Donaldson \& Lam, 2004; Watkins \& Moulds, 2005). As such, for non-depressed participants, we predicted no between-condition differences.

\section{Materials and Methods}

\section{Participants}

Participants were 86 individuals (46 females; age: $M=23.93$ years, $S D=4.73$ ) recruited from the community via advertisements placed on university noticeboards. Specifically, the recruitment flyer was worded as follows: 'Are you currently experiencing major depressive disorder or a major depressive episode? OR Have you never experienced a major depressive disorder or major depressive episode? We are currently looking for individuals who meet the criteria above to participate in a study which investigates how mood affects learning. For an hour of your time, we will reimburse you \$15.' Ethics approval was obtained from The University of New South Wales Psychology Human Research Ethics Advisory Panel C - Behavioural (approval number: 1243). Participants provided written consent to participate in the study and were reimbursed for their time (\$AUD 15 per hour). ${ }^{2}$

\section{Design}

The study was a 2 (condition; rumination, distraction) $\times 2$ (group: clinically depressed, non-depressed) between-subjects design. The main dependent variable was participants' judgment of control.

\section{Instruments}

SCID - DSM-IV - Mood Module (First, Spitzer, Gibbons, EF Williams, 1996). This semi-structured clinical interview was used to assess the current and lifetime prevalence of mood disorders. A provisionally registered psychologist administered the SCID.

Beck Depression Inventory - Second Edition (BDI-II; Beck, Steer, E Brown, 1996). The BDI-II is a 21 -item, self-report measure of depressive symptoms experienced over the last two weeks. Dozois, Dobson, and Ahnberg (1998) reported that the BDI-II possesses high internal consistency (.91) and good diagnostic discrimination. Although the original depressive realism study (Alloy \& Abramson, 1979) and other subsequent studies that have demonstrated depressive realism (e.g., Msetfi et al., 2005) employed the BDI, the BDI-II was administered in the current study in order to align with the current conceptualisation of clinical depression as defined by the Diagnostic and statistical manual of mental disorders (DSM-IV; American Psychiatric Association, 2000). The convergent validity between these two measures is very high $(r=.93, p<$ .01; Dozois et al., 1998).

Ruminative Response Scale (RRS; Nolen-Hoeksema, 1991). The RRS is a 22-item, self-report measure of the tendency to engage in rumination in response to sad mood. The RRS possesses good psychometric properties (Cronbach's alpha $=.89$ ).

Manipulation checks. The manipulation checks consisted of questions that indexed mood (happy, despondent), self-focus (focused on myself), and abstract thinking (thinking in an abstract way, trying to explain/understand things, thinking in a verbal way). Participants rated each item from $0=$ not at all, to $100=$ extremely. Participants completed the items that checked mood pre- and postinduction (in order to gauge any 


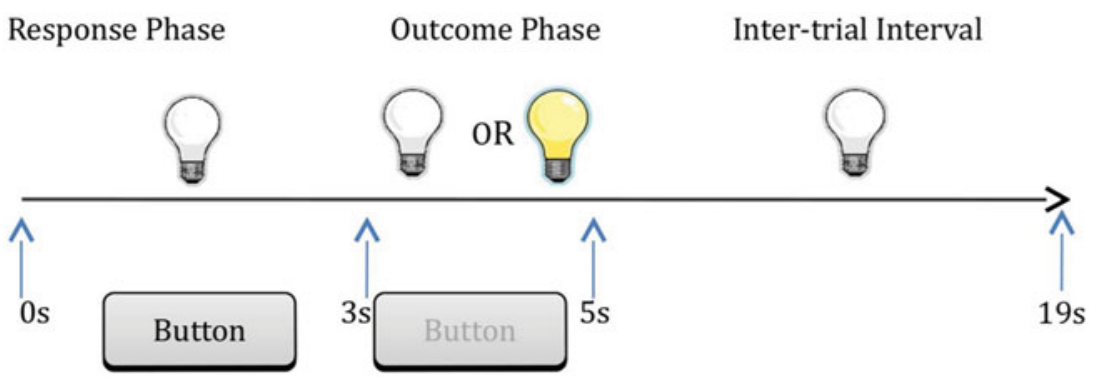

FIGURE 1

Construction of a trial in contingency task.

mood effects as a result of the manipulations), and completed the remaining checks postinduction (in order to check that the rumination manipulation induced more self-focus and abstract thinking relative to the distraction condition).

\section{Procedure}

Participants were administered the SCID-IV and BDI, followed by the premanipulation check questions and the randomly assigned induction task. The induction task consisted of 28 items, and each item appeared on the screen for 20 seconds. After reading all of the 28 items, participants completed the postmanipulation check questions. In the rumination condition, participants were asked to think about the causes, meaning and consequences of each item (e.g., the way you react, how relaxed or agitated you feel; Watkins \& Moulds, 2005). In the distraction condition participants were asked to visualise and focus their minds on each of the items (e.g., the layout of a typical classroom).

Participants then completed the contingency task, in which they were instructed to take on the role of a scientist to determine whether or not they had control over the illumination of a light. Participants were told that they should press the button on some trials, but that they should not press the button on every trial in order to see what happens on these trials. Participants were then told they would be asked questions about how much control they thought they had over the illumination of the light at the end of the experiment.

The contingency task consisted of 40 trials, and the contingency was set to zero (they had control over the illumination of the light and actual control was zero). This particular contingency task was chosen as Alloy and Abramson (1979) demonstrated the depressive realism effect in conditions in which: (1) the contingency between the response and the outcome was zero; (2) the outcome appeared on at least 50\% (or $75 \%$ ) of occasions; and (3) participants were instructed when they could and could not respond, which was then followed by an outcome or not (i.e., the contingency task had a discrete trial structure).

Participants had 3 seconds to make a response on each trial (i.e., press the button, not press the button; see Figure 1). The onset of the trial was signalled by a message on the screen: 'You may now press the button'. Then the outcome appeared on the screen for 2 seconds (light bulb in 'On' state or 'Off' state). In the ITI the light bulb went back to or remained in its off state for 15 seconds. An ITI of 15 seconds was used 
for this contingency task as it increased the chances of the depressive realism effect than (e.g., Msetfi et al., 2007; Msetfi et al., 2005). The contingency task consisted of 40 trials. Of these 40 trials, the light illuminated on 30 trials and did not illuminate on 10 trials. The presentation order of these trials was randomised for each person, independent of participants' button press responses. At the end of the task participants were asked to rate: 'How much control did your button presses have over whether the light came on?' from 0 (no control) to 100 (total control) (i.e., judgment of control).

Finally, participants completed the RRS and were debriefed and reimbursed for their time. The study took approximately one hour to complete.

\section{Analyses}

Two-way analyses of variance (ANOVAs) were conducted with the between-subject factors of condition (rumination, distraction) and group (clinically depressed, nondepressed) as the independent variables, and judgment of control as the dependent variable. In order to provide an estimate of the size of the difference between conditions, standardised 95\% confidence intervals (CIs) were constructed using the Scheffe procedure (see Bird, 2004). Standardised confidence intervals allow for an interpretation of a range of plausible effect sizes. The advantage of this procedure over obtaining a point estimate of an effect, such as eta squared, is that it provides information on the direction of the effect and the lower and upper estimates of the effect size (i.e., the width of a CI). Importantly, the width of a CI provides information about the precision of the estimate, such that a narrower $\mathrm{CI}$ indicates greater precision. The precision of an estimate is directly related to the sample size and the power of a study (e.g., the larger the sample size and power, the more precise the CI). To interpret these CIs, Cohen's d (Cohen, 1988) of .2, .5 and .8, representing a small, medium and large effect size respectively, were used.

\section{Results and Discussion}

Participants were classified as clinically depressed if they met criteria for a current major depressive episode (MDE; DSM-IV-TR, APA, 2000) and also scored in at least the mild range (i.e., $\geq 14$ ) on the BDI-II. Participants were classified as non-depressed if they did not meet criteria for a current $\mathrm{MDE}$ and they scored in the minimal range (i.e., $\leq 13$ ) on the BDI-II.

Accordingly, 28 individuals (21 females) were classified as clinically depressed (age: $M=24.79, S D=6.04$ ), and 39 individuals (18 females) were classified as nondepressed (age: $M=22.74, S D=2.85$ ). Nineteen individuals were excluded from further analyses on the basis that they did not meet the criteria of either group. The demographic details of participants are provided in Table 1.

\section{Demographic Details}

A 2 (condition: rumination, distraction) $\times 2$ (group: clinically depressed, nondepressed) between-subjects ANOVA was conducted on each of the dependent measures relevant to participants' demographic details.

For age, there were no main effects of group, $F(1,63)=3.45, p=.07$, or condition, $F<1$, and no group $\times$ condition interaction, $F<1$.

The mean and standard deviations for each condition on the instruments (BDI-II, RRS) and rumination induction manipulation check are provided in Table 2. 


\section{TABLE 1}

Demographic Details for Non-Depressed and Clinically Depressed Participants

\begin{tabular}{lll}
\hline & $\begin{array}{l}\text { Non-depressed } \\
(n=39)\end{array}$ & $\begin{array}{l}\text { Clinically dsepressed } \\
(n=28)\end{array}$ \\
\hline Gender (\% female) & 46.20 & 75.00 \\
Age (years) & $22.74(2.85)$ & $24.79(6.04)$ \\
Years of education (years) & $15.61(1.9)$ & $16.02(2.69)$ \\
Native English speakers (\%) & 33.33 & 35.71 \\
Non-native speakers: Years of English & $10.43(7.07)$ & $12.86(6.64)$ \\
Marital status (Never married \%) & 97.44 & 89.29 \\
\hline
\end{tabular}

Note: ${ }^{\text {a }}$ Standard deviations appear in parentheses.

\section{BDI- /I}

For BDI-II scores, there was an expected main effect of group, $F(1,63)=281.07$, $p<.01$, such that clinically depressed participants reported more depressive symptoms. Importantly, there was no main effect of condition, $F<1$, and no group $\times$ condition interaction, $F(1,63)=1.52, p=.22$.

\section{RRS}

For RRS scores, there was a main effect of group, $F(1,63)=43.56, p<.001$, such that clinically depressed participants reported more rumination than did non-depressed participants. There was no main effect of condition, $F<1$, and no group $\times$ condition interaction, $F<1$.

\section{Rumination Manipulation Induction Check}

A 2 (condition: rumination, distraction) $\times 2$ (group: clinically depressed, nondepressed) $\times$ (2) (time: premanipulation, postmanipulation) mixed factorial ANOVA, with repeated measures on time, was conducted on the manipulation check measures.

For happiness ratings, there was an expected main effect of group, $F(1,63)=20.95$, $p=.00$, such that non-depressed participants rated their mood as happier than did clinically depressed participants. There were no main effects of condition or time; and no group $\times$ time, condition $\times$ time, or condition $\times$ group $\times$ time interactions, all $F$ s $<1$.

For ratings of self-focus, there was a main effect of group, $F(1,63)=5.58, p=.02$, such that non-depressed participants reported higher self-focus than did clinically depressed participants. This result was unexpected, and accordingly, group was entered as a covariate to examine the main effect of condition. As expected, the main effect

114 of condition was significant, $F(1,63)=18.75, p<.01$, such that participants in the rumination condition reported higher self-focus than did participants in the distraction condition. There was no group $\times$ condition interaction, $F<1$.

For ratings of rumination thinking, there was a main effect of group, $F(1,63)=$ $6.38, p=.01$, such that clinically depressed participants were thinking in a more rumination way were non-depressed participants. This result was unexpected, and accordingly, group was entered as a covariate to examine the main effect of condition. As expected, the main effect of condition was significant, $F(1,63)=4.79, p=.03$, such 
TABLE 2

Mean BDI-II, RRS and Manipulation Check Scores

\begin{tabular}{|c|c|c|c|c|c|}
\hline & & \multicolumn{2}{|c|}{ Non-depressed ( $n=39)$} & \multicolumn{2}{|c|}{ Clinically depressed $(n=28)$} \\
\hline & & Rumination $(n=20)$ & Distraction $(n=19)$ & Rumination $(n=13)$ & Distraction $(n=15)$ \\
\hline BDI-II & & $5.05(3.71)$ & $7.47(5.66)$ & $30.84(6.26)$ & $29.73(7.54)$ \\
\hline RRS & & $38.10(14.44)$ & $42.26(12.25)$ & $61.23(10.30)$ & $59.73(11.18)$ \\
\hline \multirow[t]{2}{*}{ Happy } & Time 1 & $64.00(21.62)$ & $61.58(25.00)$ & $37.69(22.42)$ & $36.00(19.20)$ \\
\hline & Time 2 & $66.00(24.79)$ & $59.47(28.77)$ & $39.23(26.91)$ & $40.67(17.10)$ \\
\hline \multirow[t]{2}{*}{ Despondency } & Time 1 & $16.00(15.69)$ & $18.95(22.83)$ & $56.15(25.34)$ & $58.67(26.42)$ \\
\hline & Time 2 & $22.5(21.97)$ & $15.26(23.18)$ & $49.23(30.40)$ & $42.67(24.33)$ \\
\hline \multirow[t]{2}{*}{ Self-focus } & Time 1 & $65.50(23.28)$ & $62.63(17.59)$ & $60.00(28.58)$ & $48.67(24.46)$ \\
\hline & Time 2 & $83.50(10.89)$ & $54.21(33.05)$ & $66.15(27.24)$ & $40.67(30.34)$ \\
\hline \multirow[t]{2}{*}{ Abstract } & Time 1 & $41.00(25.93)$ & $39.47(26.35)$ & $71.54(26.72)$ & $58.00(28.83)$ \\
\hline & Time 2 & $53.00(30.80)$ & $32.63(29.97)$ & $66.92(32.50)$ & $56.67(27.94)$ \\
\hline Understand & Time 2 & $68.50(23.00)$ & $43.68(33.53)$ & $70.77(25.65)$ & $48.00(32.12)$ \\
\hline Verbal & Time 2 & $61.50(30.66)$ & $26.31(23.14)$ & $50.76(33.78)$ & $28.00(30.04)$ \\
\hline
\end{tabular}

Note: ${ }^{\text {a }}$ Standard deviations appear in parentheses 


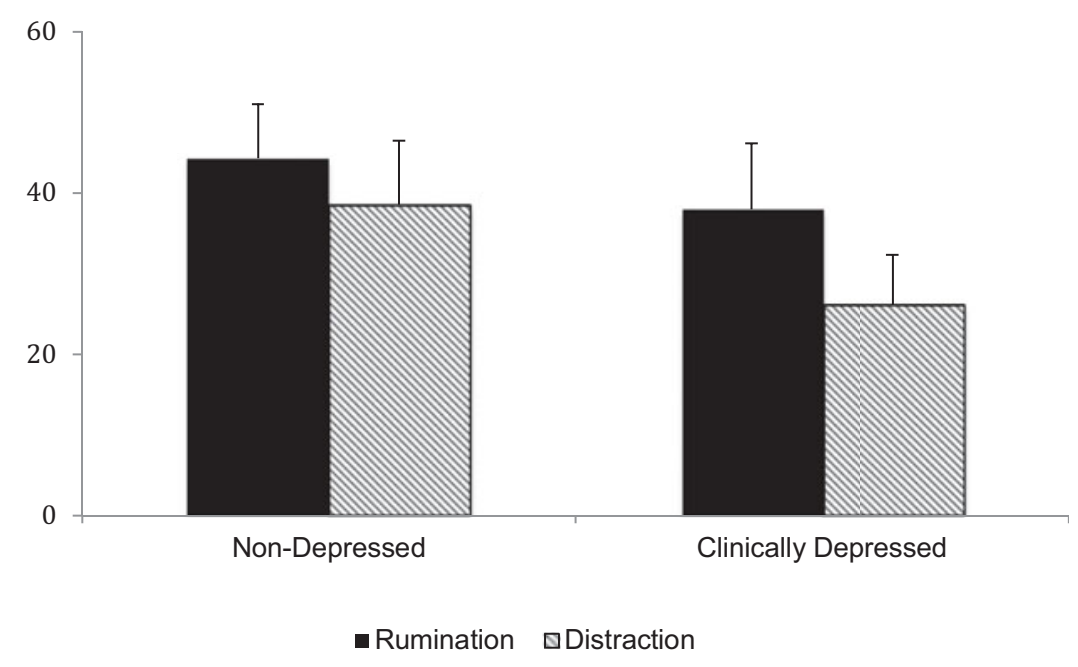

\section{FIGURE 2}

Mean judgment of control for non-depressed and clinically depressed participants, for the rumination and distraction conditions. Error bars indicate SEM.

that participants in the rumination condition were thinking in a more abstract way than those in the distraction condition. There was no group $\times$ condition interaction, $F<1$.

For ratings of verbal thinking, there was no main effect of group, $F<1$, and no group $\times$ condition interaction, $F<1$. There was a main effect of condition, $F(1,63)=15.96, p<.01$. As predicted, participants in the rumination condition reported thinking in a more verbal way than those in the distraction condition.

For ratings of trying to understand the task, there was no main effect of group, $F<$ 1 , and no group $\times$ condition interaction, $F<1$. As expected, there was a main effect of condition, $F(1,63)=11.01, p=.002$, such that participants in the rumination condition reported trying to understand the task more than those in the distraction condition.

Taken together, these analyses confirm that the rumination manipulation was successful. That is, participants in the rumination condition reported greater levels of self-focus, abstract and verbal thinking, and made a greater attempt to try to understand the task relative to those in the distraction condition.

\section{Judgment of Control}

116 Figure 2 illustrates participants' mean judgment of control. A two-way ANOVA (condition: rumination, distraction; group: clinically depressed, non-depressed) with judgment of control as the dependent variable revealed that there were no main effects of group or condition, $F(1,63)=1.54, p=.22,95 \%$ CI $[-.19,0.80]$, and $F(1,63)=1.36, p=.25,95 \%$ CI $[-.21,0.79]$, respectively. There was no group $\times$ condition interaction, $F<1$. Although numerically clinically depressed individuals provided lower judgments of control than non-depressed individuals, this difference was not statistically different. Thus, the depressive realism effect was not replicated. 
The confidence interval provides a range of plausible population effect sizes and is constructed on the basis of the observed effect within our experimental sample. The confidence intervals indicate that the size of the effect of group on the judgment of control ranged from a small negative effect (i.e., - .19; non-depressed individuals having more accurate judgments of control than clinically depressed individuals) to a large positive effect (i.e., .80; clinically depressed individuals having more accurate judgments than non-depressed individuals). Interestingly, although numerically participants in the rumination condition provided higher judgments of control than those in the distraction condition, there was no statistically significant difference between the conditions. The size of the effect of condition on the judgment of control ranged from a small negative effect (i.e., -.21 ; individuals in the distraction condition having more accurate judgments of control than those in the rumination condition) to a large positive effect (i.e., .79; individuals in the rumination condition having more accurate judgments of control than those in the distraction condition).

Bayesian analysis was conducted to determine the strength of evidence for the null hypothesis. For the main effect of group, the Bayes factor was 1.29, and for the main effect of condition, the Bayes factor was 1.18 and suggests that there is anecdotal evidence in favour of the alternative hypothesis. For the group $\times$ condition interaction, the Bayes factor was 0.65 and suggests that there is anecdotal evidence in favour of the null hypothesis.

\section{Rate of Responding and Experienced Contingency}

One explanation of the depressive realism effect is that depressed participants respond less frequently than non-depressed participants (Blanco et al., 2009). Specifically, it has been theorised that the lower rate of responding by depressed individuals may lead them to experience a lower contingency. According to this account, as a result of less frequent responding, the relative number of a and b cells (i.e., trials on which a response is made) decrease and the number of $c$ and $d$ cells (i.e., trials on which no response is made) increase. Since objective contingency is: $a /(a+b)-c /(c+d)$, fewer $\mathrm{a}$ and $\mathrm{b}$ cells and more $\mathrm{c}$ and $\mathrm{d}$ cells leads to a negative contingency. As a result, the experience of a lower contingency leads to lower judgments of control than non-depressed participants.

In order to test Blanco et al.'s hypothesis, response rates and the contingency experienced between conditions and groups are reported. Two-way ANOVAs were conducted with the between-subject factors of condition (rumination, distraction) and group (clinically depressed, non-depressed), with response rate and experienced contingency as the dependent variables. The mean response rates for non-depressed rumination and distraction conditions were $M=53.88(S D=16.65)$ and $M=$ $59.74(S D=22.97)$ respectively. The mean response rates for clinically depressed rumination and distraction conditions were $M=50.77(S D=17.12)$ and $M=53.17$ $(S D=19.17)$ respectively. For response rate, the main effects of group and condition were not significant, $F(1,63)=1.02, p=.32$, and $F<1$ respectively. There was no group $\times$ condition interaction, $F<1$.

The mean experienced contingency for non-depressed rumination and distraction conditions were $M=-0.02(S D=0.15)$ and $M=0.13(S D=0.31)$ respectively. The mean experienced contingency for clinically depressed rumination and distraction conditions were $M=-0.01(S D=0.12)$ and $M=-0.05(S D=0.14)$ respectively. For experienced contingency the main effects of group and condition were not significant, 
$F(1,63)=2.79, p=.10$, and $F(1,63)=1.18, p=.28$, respectively. There was a significant group $\mathrm{x}$ condition interaction, $F(1,63)=4.21, p=.04$. This result was unexpected, and follow-up independent $t$ tests demonstrated that there was no difference between the clinically depressed and non-depressed participants in the rumination condition, $t(31)=-.39, p=.35$. However, in the distraction condition, there was an effect of group, $t(32)=2.17, p=.02$, such that clinically depressed participants experienced a lower contingency than did non-depressed participants. It is possible that clinically depressed participants' experience of a lower contingency may have led to lower (albeit not significantly lower) judgments of control compared to non-depressed participants. Such an explanation is consistent with Blanco et al.'s hypothesis that a lower response rate and lower experience of contingency can lead to more accurate judgments of control.

\section{Illusion of Control}

Non-depressed participants' mean judgments of control in the rumination and distraction conditions were significantly greater than zero, $F(1,63)=42.32, p<.001$, $95 \%$ CI $[1.42,3.62]$, and $F(1,63)=30.46, p<.001,95 \%$ CI $[1.07,3.32]$, respectively. Clinically depressed participants' mean judgments of control in the rumination and distraction conditions were also significantly greater than zero, $F(1,63)=20.25$, $p<.01,95 \%$ CI $[0.80,3.52]$, and $F(1,63)=11.04, p<.001,[0.22,2.75]$, respectively. Thus, all groups and conditions displayed an illusion of control. In addition, non-depressed participants in the rumination and distraction conditions provided judgments of control that were significantly greater than their experienced contingency, $F(1,63)=42.48, p<.001,95 \% \mathrm{CI}[1.43,3.62]$, and $F(1,63)=30.33, p<.001$, $95 \%$ CI [1.06, 3.31], respectively. Clinically depressed participants in the rumination and distraction conditions also provided judgments of control that were significantly greater than their experienced contingency, $F(1,63)=20.31, p=.00,95 \% \mathrm{CI}[0.81$, $3.52]$, and $F(1,63)=11.11, p=.001,95 \% \mathrm{CI}[0.23,2.75]$, respectively. Thus, in all groups and conditions, participants displayed an illusion of control. In addition, the effect size of the illusion of control was small for clinically depressed participants in the distraction condition (i.e., .22), and at least large for the remaining cells (i.e., .80).

\section{Judgment of Control (Extreme Responders Excluded)}

As previously outlined, one potential explanation of the depressive realism effect is that a lower rate of responding by depressed individuals may lead them to provide more accurate judgments of control (Blanco et al., 2009). However, this greater accuracy may not necessarily be due to their ability to calculate contingency accurately. While the analysis of response rate between conditions was not significant for this study, it is possible that within each condition there was significant variation in participants' responses such that some participants made very few responses while others made many.

118 It is plausible that this variation may have potentially masked differences between the conditions on participants' judgment of control. Thus, another test of whether clinical depression leads to greater accuracy, independent of response rate, would be to remove extreme responders (R. Murphy, personal communication, September 14, 2010). Such an analysis would remove further noise from the data. One way to remove extreme responders is to exclude participants if they respond more than one standard deviation away from (i.e., greater or less than) the mean number of responses. This exclusion criterion was chosen on the basis that participants were explicitly instructed 
to respond on approximately half of the trial presentations (i.e., 20 trials). Therefore, a participant who responded within one standard deviation of the mean provided a strong indication of compliance with the experimental instructions, whereas a participant who responded within two standard deviations of the mean indicated potential non-compliance with the instructions. Such non-compliance justified exclusion of these extreme responders. The mean number of responses was 21.91 (SD = 7.65). Accordingly, participants were excluded if they responded outside the range of 14.2629.56. This resulted in the exclusion of 19 participants (rumination non-depressed: $n=4$; distraction non-depressed: $n=7$; rumination clinically depressed: $n=3$; distraction clinically depressed: $n=5$ ). The mean judgments of control for included non-depressed participants in the rumination and distraction conditions were $M=$ $38.75(S D=38.75)$ and $M=29.08(S D=29.08)$ respectively. The mean judgments of control for included clinically depressed participants in the rumination and distraction conditions were $M=44.00(S D=28.38)$ and $M=26.00(S D=27.04)$ respectively. A two-way ANOVA with the factors of experimental condition (rumination, distraction) and group (clinically depressed, non-depressed) was conducted. There were no main effects of group or condition, $F<1,95 \%$ CI $[-.63, .57]$, and $F(1,43)=2.49, p=$ $.12,95 \% \mathrm{CI}[-.13,1.06]$, respectively. There was no group $\times$ condition interaction, $F<1$. Thus, the depressive realism effect was not replicated when extreme responders were removed. The size of the effect of group on the judgment of control ranged from a medium negative effect (i.e., -.63 ; non-depressed individuals having more accurate judgments of control than clinically depressed individuals) to a medium positive effect (i.e., .57; clinically depressed individuals having more accurate judgments than non-depressed individuals). The size of the effect of condition on the judgment of control ranged from a small negative effect (i.e., -.13 ; individuals in the distraction condition having more accurate judgments of control than those in the rumination condition) to a large positive effect (i.e., 1.06; individuals in the rumination condition having more accurate judgments of control than those in the distraction condition).

Contrary to our hypotheses, participants in the clinically depressed group did not demonstrate more accurate judgments of control than those in the non-depressed group. This study, to the best of our awareness, is the first study to measure rumination (i.e., via the RRS) and manipulate rumination with a clinically depressed and nondepressed sample. However, participants in the rumination condition did not provide more accurate ratings of judgment of control than participants in the distraction condition. Not only was the depressive realism effect not replicated, these results are inconsistent with the ITI hypothesis, which proposes that depressed individuals may engage in rumination during the ITI and consequently provide more 'accurate' judgments of control.

Despite the fact that we attempted to capitalise on conditions that are theorised to produce the effect (i.e., employing a clinically depressed sample and inducing rumination), we did not replicate the depressive realism effect. This non-replication is in fact consistent with the findings of Moore and Fresco's (2012) meta-analytic review showing that the depressive realism is difficult to replicate and, further, that when it is replicated, it yields a conventional small effect. Rather, in the current study, the illusion of control was observed in both the depressed and non-depressed groups, and thus appears to be difficult to attenuate. These findings are at odds with the original Alloy and Abramson (1979) study in which they found that depressed individuals were more realistic than their non-depressed counterparts. 
There are several potential reasons as to why rumination may not have decreased judgments of control for participants in the rumination condition. One potential reason is that participants were not specifically instructed to ruminate during the ITI. Rather, the rumination manipulation was administered prior to participants completing the contingency task. The administration of the rumination manipulation prior to a task replicates the standard experimental approach in the rumination literature of inducing rumination and examining its effect on a subsequent task (e.g., generation of solutions for scenarios in a social problem-solving task; Watkins \& Teasdale, 2004). However, the ITI hypothesis specifically proposes that rumination that occurs during the ITI may be responsible for the depressive realism effect. In order to more directly test the central tenets of the ITI hypothesis, the administration of a rumination induction during the ITI, or at the very least taking a measurement of the degree of participants' rumination during this period, may be a better approach for researchers to take in future work. Indeed, we considered this approach during the process of designing this study, but opted not to employ such a method owing to concerns about the feasibility and practicality of inducing rumination repeatedly during the brief ITI periods. Instead, we employed the standard experimental induction procedures. Alternatively, or perhaps in addition, in future studies participants could be asked to rate the extent to which they are engaging in rumination during the ITI immediately after the completion of the contingency task. If depressed individuals' ratings were higher than those of non-depressed participants, in the context of replicating the depressive realism effect, this would provide evidence for the ITI hypothesis.

Another reason that rumination may not have lowered clinically depressed participants' judgments of control may be that the contingency task used in this experiment did not contain negative, self-referential stimuli. The literature on information processing biases in depression suggests that there are at least two important conditions that are required in order to observe information processing biases in depression; specifically: (a) the stimuli in the task needs to be negative and self-referential, and (b) there needs to be sufficient time in the task to engage in elaborative processing (or rumination) (Wisco, 2009). While the experimental task provided sufficient time to engage in elaborative processing, it did not contain self-referential stimuli. We therefore cannot rule out the possibility that if the contingency task had been comprised of negative, self-referential stimuli (e.g., for the outcome), depressed participants may have provided lower (and more accurate) judgments of control. That said, we employed the same procedure as Msetfi et al. (2007) and Msetfri et al. (2005), who did not utilise negative self-referential stimuli, yet replicated the depressive realism effect.

Given the robustness of the illusion of control demonstrated in the findings of the present study, it may be possible that a different version of the contingency task may increase the likelihood of attenuating the illusion of control; for example, if the outcome of the contingency task was changed from the illumination of a light to the initiation of a smile from a stranger and participants were asked to determine the extent to which they could control the occurrence of a smile by either giving a compliment or not (i.e., equivalent to pressing or not pressing the button). This modified task may elicit mood related cognitive processes.

\section{Conclusion}

Our study is one of the few to have examined the depressive realism effect in a clinically depressed sample and the only study to experimentally manipulate rumination. 
The present study clearly demonstrates the robustness of the illusion of control. Indeed, one of the main aims of treatment for depression is to improve an individual's internal sense of control. Hence, an understanding of how to modulate the illusion of control - particularly when there are negative, self-referential stimuli that engage a depressed individual's maladaptive cognitive processes - may assist in potentially improving treatments of depression. That is, treatment efficacy may be improved through a greater understanding of the factors that promote an individuals sense of self-efficacy.

\section{Acknowledgments}

The study reported in this article was conducted as part of the first author's doctoral dissertation. This study was supported by an Australian Postgraduate Award (APA) to the first author.

\section{Endnotes}

1 In this study, participants were categorised as depressed if they scored above 9 on the BDI (Beck Ward, Mendelson, Mock, \& Erbaugh, 1961), and as non-depressed if they scored less than 9 on the BDI.

2 Previous depressive realism studies have typically included a sample size of approximately 75 participants (e.g., Msetfi et al., 2007). Consequently, the sample size in our study is consistent with studies in the existing literature. In order to provide more information on the size of the effect and the direction of the effect, confidence intervals were constructed and are reported, to the $F$ value.

\section{References}

Allan, L.G. (1980). A note on measurement of contingency between two binary variables in judgment tasks. Bulletin of the Psychonomic Society, 15, 147-149.

Alloy, L.B., \& Abramson, L.Y. (1979). Judgment of contingency in depressed and nondepressed students: Sadder but wiser? Journal of Experimental Psychology: General, 108, 441-485.

American Psychiatric Association. (2000). Diagnostic and statistical manual of mental disorders (4th ed., text rev.). Washington, DC: Author.

Beck, A.T. (1967). Depression: Clinical, experimental and theoretical aspects. New York, NY: Harper \& Row.

Beck, A.T., Rush, A.J., Shaw, B.F., \& Emery, G. (1979). Cognitive therapy of depression. New York, NY: Guilford.

Beck, A.T., Steer, R.A., \& Brown, G.K., (1996). Beck Depression Inventory — Second Edition manual. San Antonio, TX: The Psychological Corporation.

Beck, A.T., Ward, C.H., Mendelson, M., Mock, J., \& Erbaugh, J. (1961). An inventory for measuring depression. Archives of General Psychiatry, 4, 561-571.

Bird, K.D. (2004). Analysis of variance via confidence intervals. London: Sage Publications.

Blanco, F., Matute, H., \& Vadillo, M.A. (2009) Depressive realism: Wiser or quieter? The Psychological Record, 59, 551-562.

Donaldson, C., \& Lam, D., (2004). Rumination, mood, and social problem-solving in major depression. Psychological Medicine, 34, 1309-1318.

Dozois, D.J., Dobson, K.S., \& Ahnberg, J.L. (1998). A psychometric evaluation of the Beck Depression Inventory - II. Psychological Assessment, 10, 83-89.

First, M.B., Spitzer, R.L., Gibbons, M., \& Williams, J.B.W. (1996). Structured Clinical Interview for DSM - IV Axis I Disorders (SCID - I). Washington, DC: American Psychiatric Press. 
Lyubomirsky, S., Caldwell, N.D., \& Nolen-Hoeksema, S. (1998). Effects of ruminative and distracting responses to depressed mood on retrieval of autobiographical memories. Journal of Personality and Social Psychology, 75, 166-177.

Lyubomirsky, S., \& Nolen-Hoeksema, S. (1995). Effects of self — Focused rumination on negative thinking and interpersonal problem solving. Journal of Personality and Social Psychology, 69, 176190.

Moore, M.T., \& Fresco, D.M. (2012). Depressive realism: A meta-analytic review. Clinical Psychology Review, 32, 496-509.

Msetfi, R.M., Murphy, R.A., \& Simpson, J. (2007). Depressive realism and the effect of intertrial interval on judgments of zero, positive and negative contingencies. Quarterly Journal of Experimental Psychology, 60, 461-481.

Msetfi, R.M., Murphy, R.A., Simpson, J., \& Kornbrot, D.E. (2005). Depressive realism and outcome density bias in contingency judgments: The effect of the context and intertrial interval. Journal of Experimental Psychology: General, 134, 10-22.

Nolen-Hoeksema, S. (1991). Responses to depression and their effects on the duration of depressive episodes. Journal of Abnormal Psychology, 100, 569-582.

Nolen-Hoeksema, S., Morrow, J., \& Fredrickson, B.L. (1993). Response styles and the duration of episodes of depressed mood. Journal of Abnormal Psychology, 102, 20-28.

Watkins, E., \& Teasdale, J.D. (2004) Adaptive and maladaptive self-focus in depression. Journal of Affect Disorders, 82, 1-8.

Watkins, E.D., \& Moulds, M.L., (2005). Distinct modes of ruminative self-focus: Impact of abstract versus concrete rumination on problem solving in depression. Emotion, 5, 319-328.

Wisco, B.E. (2009). Depressive cognition: Self-reference and depth of processing. Clinical Psychology Review, 29, 382-392. 